\title{
INHOMOGENEOUS PERIODIC PARABOLIC PROBLEMS WITH INDEFINITE DATA
}

\author{
T. GODOY and U. KAUFMANN ${ }^{凶}$
}

(Received 7 May 2011)

\begin{abstract}
Let $\Omega \subset \mathbb{R}^{N}$ be a smooth bounded domain and let $f \not \equiv$ be a possibly discontinuous and unbounded function. We give a necessary and sufficient condition on $f$ for the existence of positive solutions for all $\lambda>0$ of Dirichlet periodic parabolic problems of the form $L u=h(x, t, u)+\lambda f(x, t)$, where $h$ is a nonnegative Carathéodory function that is sublinear at infinity. When this condition is not fulfilled, under some additional assumptions on $h$ we characterize the set of $\lambda$ s for which the aforementioned problem possesses some positive solution. All results remain true for the corresponding elliptic problems.
\end{abstract}

2010 Mathematics subject classification: primary 35K20; secondary 35P05, 35B10.

Keywords and phrases: periodic parabolic problems, indefinite, sub- and supersolutions, elliptic problems.

\section{Introduction}

Let $\Omega$ be a $C^{2+\theta}$ bounded domain in $\mathbb{R}^{N}, \theta \in(0,1), N \geq 2$. For $T>0$ and $1 \leq p \leq \infty$, let $L_{T}^{p}$ be the Banach space of $T$-periodic functions $g$ on $\Omega \times \mathbb{R}$ (that is, satisfying $g(x, t)=$ $g(x, t+T)$ a.e., $(x, t) \in \Omega \times \mathbb{R})$ such that $g_{\mid \Omega \times(0, T)} \in L^{p}(\Omega \times(0, T))$, equipped with the norm $\|g\|_{L_{T}^{p}}:=\left\|g_{\mid \Omega \times(0, T)}\right\|_{L^{p}(\Omega \times(0, T))}$. Let $C_{T}^{1+\theta,(1+\theta) / 2}$ and $C_{T}$ be the spaces of $T$-periodic functions on $\bar{\Omega} \times \mathbb{R}$ belonging to $C^{1+\theta,(1+\theta) / 2}(\bar{\Omega} \times \mathbb{R})$ and $C(\bar{\Omega} \times \mathbb{R})$ respectively, and let

$$
P^{\circ}:=\text { the interior of the positive cone of } C_{T}^{1+\theta,(1+\theta) / 2} \text {. }
$$

Let $\left\{a_{i j}\right\},\left\{b_{j}\right\}, 1 \leq i, j \leq N$, be two families of $T$-periodic functions satisfying $a_{i j} \in C^{0,1}(\bar{\Omega} \times \mathbb{R}), b_{j} \in L_{T}^{\infty}, a_{i j}=a_{j i}$ and

$$
\sum a_{i j}(x, t) \varsigma_{i} \varsigma_{j} \geq \alpha \mid \varsigma^{2}
$$

for some $\alpha>0$ and all $(x, t) \in \Omega \times \mathbb{R}, \varsigma \in \mathbb{R}^{N}$. Let $A$ be the $N \times N$ matrix whose $i, j$ entry is $a_{i j}$, let $\bar{b}=\left(b_{1}, \ldots, b_{N}\right)$, let $0 \leq c_{0} \in L_{T}^{r}, r>N+2$, and let $L$ be the parabolic operator given by

$$
L u:=u_{t}-\operatorname{div}(A \nabla u)+\langle\bar{b}, \nabla u\rangle+c_{0} u .
$$

The research was partially supported by Secyt-UNC and ANPCYT.

(C) 2011 Australian Mathematical Publishing Association Inc. 0004-9727/2011 \$16.00 
Let $W:=\left\{u \in L^{2}\left((0, T), H_{0}^{1}(\Omega)\right): u_{t} \in L^{2}\left((0, T), H^{-1}(\Omega)\right)\right\}$ and $g \in L_{T}^{2}$. We say that $u$ is a (weak) solution of the Dirichlet periodic problem

$$
\begin{cases}L u=g & \text { in } \Omega \times \mathbb{R} \\ u=0 & \text { on } \partial \Omega \times \mathbb{R} \\ u T \text {-periodic } & \end{cases}
$$

if $u$ is $T$-periodic, $u_{\mid \Omega \times(0, T)} \in W$ and

$$
\int_{\Omega \times(0, T)}\left[-u \phi_{t}+\langle A \nabla u, \nabla \phi\rangle+\langle\bar{b}, \nabla u\rangle \phi+c_{0} u \phi\right]=\int_{\Omega \times(0, T)} g \phi
$$

for all $\phi \in C_{c}^{\infty}\left(\Omega \times \mathbb{R}\right.$ ) (and so for all $\phi \in L_{T}^{\infty}$ such that $\phi_{\mid \Omega \times(0, T)} \in V_{0}$, where $V_{0}:=$ $\left.L^{2}\left((0, T), H_{0}^{1}(\Omega)\right)\right)$. For $u \in W$, the inequality $L u \geq g$ (respectively $\leq$ ) will be understood in the same sense.

Let $\widetilde{W}:=\left\{u \in L^{2}\left((0, T), H^{1}(\Omega)\right): u_{t} \in L^{2}\left((0, T), H^{-1}(\Omega)\right)\right\}$. Following [13], we say that $v$ is a supersolution of $(1.1)$ if $v_{\mid \Omega \times(0, T)} \in \widetilde{W}, v_{t} \in L^{2}\left((0, T), H^{-1}(\Omega)\right)+L^{1+\eta}(\Omega \times$ $(0, T))$ for $\eta>0$ small enough, $v_{\mid \partial \Omega \times(0, T)} \geq 0, v(\cdot, 0)=v(\cdot, T)$ a.e. in $\Omega$ and

$$
\int_{\Omega \times(0, T)}\left[-v \phi_{t}+\langle A \nabla v, \nabla \phi\rangle+\langle\bar{b}, \nabla v\rangle \phi+c_{0} v \phi\right] \geq \int_{\Omega \times(0, T)} g \phi
$$

for all $0 \leq \phi \in C_{c}^{\infty}\left(\Omega \times(0, T)\right.$ ) (and so for all $0 \leq \phi \in L_{T}^{\infty}$ such that $\phi_{\mid \Omega \times(0, T)} \in V_{0}$ with $V_{0}$ as above). A subsolution is similarly defined by reversing the above inequalities.

For $1 \leq r \leq \infty$ let $W_{r}^{2,1}\left(\Omega \times\left(t_{0}, t_{1}\right)\right)$ be the Sobolev space of the functions $u \in$ $L^{r}\left(\Omega \times\left(t_{0}, t_{1}\right)\right), u=u(x, t), x=\left(x_{1}, \ldots, x_{N}\right)$ such that $u_{t}, u_{x_{j}}$ and $u_{x_{i} x_{j}}$ belong to $L^{r}\left(\Omega \times\left(t_{0}, t_{1}\right)\right)$ for $1 \leq i, j \leq N$, and let $W_{r, T}^{2,1}$ be the space of $T$-periodic functions such that $u_{\mid \Omega \times(0, T)} \in W_{r}^{2,1}(\Omega \times(0, T))$. For $g: \Omega \times \mathbb{R} \rightarrow \mathbb{R}$ and $r>N+2$ we say that $v \in W_{r, T}^{2,1}$ is a (strong) solution of (1.1) if the equation holds a.e. in the pointwise sense.

Let $h: \Omega \times \mathbb{R} \times[0, \infty) \rightarrow[0, \infty)$ be a Carathéodory function, that is $(x, t) \rightarrow$ $h(x, t, \xi)$ is measurable for all $\xi \geq 0$ and $\xi \rightarrow h(x, t, \xi)$ is continuous in $[0, \infty)$ a.e., $(x, t) \in \Omega \times \mathbb{R}$. Assume that $h(\cdot, \xi)$ belongs to $L_{T}^{r}, r>(N+2) / 2$, for all $\xi \geq 0$ and that $h$ is $T$-periodic in $t$.

Our aim in this paper is to study the existence and nonexistence of positive solutions for periodic parabolic problems of the form

$$
\begin{cases}L u=h(x, t, u)+\lambda f(x, t) & \text { in } \Omega \times \mathbb{R} \\ u=0 & \text { on } \partial \Omega \times \mathbb{R} \\ u T \text {-periodic } & \end{cases}
$$

where $0 \not \equiv f \in L_{T}^{r}$ for some $r>(N+2) / 2, \lambda>0$ is a real parameter and $h$ is a nonnegative Carathéodory function that is sublinear at infinity. Let us mention that as a consequence of our proofs all the results remain true for the corresponding elliptic problems. For applications we refer to $[5,12,20]$. 
To be more precise, let:

(H1) there exist $p \in(0,1), \bar{\xi} \geq 0$ and $0 \leq a \in L_{T}^{r}$ with $r>(N+2) / 2$ and $a \not \equiv 0$, such that

$$
h(x, t, \xi) \leq a(x, t) \xi^{p} \quad \text { for all } \xi \in[\bar{\xi}, \infty) \text { a.e., }(x, t) \in \Omega \times \mathbb{R}
$$

(H2) $h(\cdot, 0)=0$, and there exist $q \in(0,1), \underline{\xi}>0$ and $0 \leq b(x, t) \not \equiv 0$, such that

$$
h(x, t, \xi) \geq b(x, t) \xi^{q} \quad \text { for all } \xi \in[0, \underline{\xi}] \text { a.e., }(x, t) \in \Omega \times \mathbb{R} ;
$$

(H3) $h(\cdot, 0)=0$, and there exist $\xi>0$ and $0 \leq b \in L_{T}^{r}$ with $r>N+2$ and $\lambda_{1}(b)<1$, such that

$$
h(x, t, \xi) \geq b(x, t) \xi \quad \text { for all } \xi \in[0, \underline{\xi}] \text { a.e., }(x, t) \in \Omega \times \mathbb{R}
$$

$\left(\lambda_{1}(b)\right.$ denotes the unique positive principal eigenvalue with respect to the weight $b$; see Remark 2.2 below).

Let us denote by $L^{-1}$ the solution operator of (1.1). If $h$ satisfies (H1), we shall prove in Theorem 3.1 that (1.2) has a nontrivial solution $0 \leq u_{\lambda} \in C_{T}$ for all $\lambda>0$ if and only if $L^{-1} f \geq 0$, and that if $L^{-1} f>0$ or $L^{-1} f \in P^{\circ}$ and $h(\cdot, \xi) \in L_{T}^{r}$ for some $r>N+2$ and all $\xi \geq 0$, then $u_{\lambda}$ can be chosen such that $u_{\lambda}>0$ or $u_{\lambda} \in W_{r, T}^{2,1} \cap P^{\circ}$ respectively.

Suppose now that $L^{-1} f \nsupseteq 0$. When either (H2) or (H3) is also fulfilled we shall see that there exist some $\underline{\Lambda}>0$ and $\Lambda \in[\underline{\Lambda}, \infty)$ such that (1.2) has a solution $0<u_{\lambda} \in C_{T}$ for all $\lambda \in(0, \underline{\Lambda}]$ and that there is no nonnegative solution for (1.2) if $\lambda>\Lambda$ (see Theorem 3.2(i)). If in addition either $f \leq 0$ or $\xi \rightarrow h(\cdot, \xi)$ is concave for all $\xi \geq 0$, then we shall show the existence of some nontrivial solution $0 \leq u_{\lambda} \in C_{T}$ for all $\lambda \in(0, \Lambda]$. Furthermore, when $f \leq 0$ we shall provide an upper bound for $\Lambda$, and in the case where $\xi \rightarrow h(\cdot, \xi)$ is concave then we shall see that $u_{\lambda}$ can be chosen such that $u_{\lambda}>0$ for all $\lambda \in(0, \Lambda)$ (see Theorem 3.2(ii)). In fact, in Remark 3.3 we show that the last assertion holds without any assumptions on the sign of $L^{-1} f$. Also, if $f$ and $h(\cdot, \xi)$ belong to $L_{T}^{r}$ for some $r>N+2$ and all $\xi \geq 0$, then we establish that $u_{\lambda} \in W_{r, T}^{2,1}$ whenever such $u_{\lambda}$ exists (in particular, $u_{\lambda} \in P^{\circ}$ whenever $u_{\lambda}>0$ ). Let us finally point out that it can be proved in some cases that the condition $\lambda_{1}(b)<1$ in (H3) is necessary for the existence of nonnegative solutions for (1.2) when $f \leq 0$ in $\Omega \times \mathbb{R}$ (see Remark 3.4 below). These results will be obtained using the well-known sub- and supersolutions method combined with some facts concerning linear problems with weight.

In order to relate our theorems to others in the literature, let us mention that similar results to those in Theorem 3.1 can be found for the corresponding elliptic problem in [10, Theorem 1.7] (see also [8]), for $L=-\Delta, h(x, \xi)=\xi^{q}(0<q<1)$ and $f \in C^{1}(\bar{\Omega}) \backslash\{0\}$, and an extension for the $p$-Laplace operator is stated in [9]. In the parabolic case, the existence of positive periodic solutions for some similar problems with $f \geq 0$ in $\Omega \times \mathbb{R}$ is treated in [17], but the approach used there fails if $f<0$ in a set of positive measure and to our knowledge no results are known in that case. We refer to [4, Section 5], [21], [18, 19] and its references for related periodic parabolic problems. 
On the other hand, analogous elliptic problems with $f \leq 0$ (alternatively referred to in the literature as nonpositone or semipositone) and $h(x, \xi)=h(\xi)$ have been studied by several authors. When $\Omega$ is a ball, $L=-\Delta, f$ is a negative constant and $h(\xi)$ is a concave function satisfying some additional conditions, it was proved with variational and symmetry arguments that there exist $0<\Lambda_{1}<\Lambda_{2}<\infty$ such that there are exactly two positive solutions for $\lambda \in\left[\Lambda_{1}, \Lambda_{2}\right)$, exactly one for $\lambda \in\left(0, \Lambda_{1}\right)$ and $\lambda=\Lambda_{2}$, and no positive solutions for $\lambda>\Lambda_{2}$ (see, for example, [24, Theorem 6.16] and [6]). For general domains (and also $L=-\Delta$ and $f$ a negative constant), existence/nonexistence and uniqueness/multiplicity of positive solutions have been considered, for instance, in $[7,11]$. We refer the reader to the survey paper [5] for more details.

Let us finally mention some literature in the general case $h=h(x, \xi)$. For a nonselfadjoint operator, semipositone problems as above with Neumann or Robin boundary conditions were considered in [2], while analogous Dirichlet superlinear problems were treated in [3]. On the other hand, many papers studying elliptic reaction diffusion equations with harvesting (which lead to nonlinearities satisfying $(\mathrm{H} 1)$ and (H3) or closely related conditions) have appeared recently; see, for example, [1, 14] and the references therein.

\section{Preliminaries}

The following two remarks give some necessary facts about the solution operator of (1.1) (denoted by $L^{-1}$ ) and periodic parabolic problems with weight.

REMARK 2.1. (i) For $r>(N+2) / 2$ we have that $L^{-1}\left(L_{T}^{r}\right) \subset C_{T}$ and $L^{-1}: L_{T}^{r} \rightarrow C_{T}$ is a compact and positive operator (see, for example, [16, Remarks 2.1 and 2.2]).

(ii) Given $g \in L_{T}^{r}, r>N+2$, there exists a unique solution $u \in W_{r, T}^{2,1}$ of (1.1) and the operator $L^{-1}: L_{T}^{r} \rightarrow W_{r, T}^{2,1}$ is continuous (see, for example, [23, Section 4]). Moreover, from the Sobolev imbedding theorems (for example, [22, Lemma 3.3, p. 80]) it follows that $u \in C_{T}^{1+\theta,(1+\theta) / 2}$; and if $g \geq 0$, the strong maximum principle (as stated, for example, in [12, Theorem 13.5]) yields that $u>0$ in $\Omega \times \mathbb{R}$ and $\partial u / \partial \nu<0$ on $\partial \Omega \times \mathbb{R}$, where $v$ denotes the outward unit normal to $\partial \Omega$. In particular, $u \in P^{\circ}$ and the boundary and periodicity conditions in (1.1) are satisfied pointwise.

Remark 2.2. Let $b \in L_{T}^{r}$ with $r>(N+2) / 2$, and let $P(b):=\int_{0}^{T} \operatorname{esssup}_{x \in \Omega} b(x, t) d t$.

(i) Then $P(b)>0$ is necessary and sufficient for the existence of a (unique and simple) positive principal eigenvalue $\lambda_{1}(b)$ for the problem

$$
\begin{cases}L \varphi=\lambda b \varphi & \text { in } \Omega \times \mathbb{R} \\ \varphi=0 & \text { on } \partial \Omega \times \mathbb{R} \\ \varphi T \text {-periodic } & \end{cases}
$$

(see [15, Theorem 3.6]). We note that $P(b)=+\infty$ is allowed (see [15, p. 218]). If $\lambda_{1}(b)$ exists, we will denote by $\varphi$ the positive principal eigenfunction normalized by $\|\varphi\|_{\infty}=1$. We have that $\varphi \in C_{T}$ and $\varphi>0$ in $\Omega \times \mathbb{R}$ (see [16, Lemma 2.3, Corollary 2.12]), and if $r>N+2$ then, from Remark 2.1, $\varphi \in W_{r, T}^{2,1} \cap P^{\circ}$. 
(ii) Suppose that $P(b)>0$ and let $\lambda \in\left(0, \lambda_{1}(b)\right)$. Then $(L-\lambda a)^{-1}: L_{T}^{r} \rightarrow C_{T}$ is a well-defined compact and positive operator (see [16, Lemma 2.9] and [15, Theorem 3.6]). In particular, if $L u>\lambda b u$ (respectively <) with $u \geq 0, u T$-periodic and $u=0$ on $\partial \Omega \times \mathbb{R}$, then $\lambda<\lambda_{1}(b)$ (respectively $\lambda>\lambda_{1}(b)$ ).

\section{Main results}

Theorem 3.1. Let h satisfy $(H 1)$ and let $0 \not \equiv f \in L_{T}^{r}$ for some $r>(N+2) / 2$. Then (1.2) has a nontrivial solution $0 \leq u_{\lambda} \in C_{T}$ for all $\lambda>0$ if and only if $L^{-1} f \geq 0$. Moreover, if $L^{-1} f>0$ or $L^{-1} f \in P^{\circ}$ and $h(\cdot, \xi) \in L_{T}^{r}$ for some $r>N+2$ and all $\xi \geq 0$, then $u_{\lambda}$ can be chosen such that $u_{\lambda}>0$ or $u_{\lambda} \in W_{r, T}^{2,1} \cap P^{\circ}$ respectively.

Proof. Let $\bar{\xi}, a(x, t)$ and $p$ be given by (H1). For $\lambda>0$, let $0 \leq \Psi=\Psi_{\lambda} \in C_{T}$ be the solution of (1.1) with $a+\lambda f^{+}$in place of $g$, where as usual we write $f=f^{+}-f^{-}$ with $f^{+}=\max (f, 0)$ and $f^{-}=\max (-f, 0)$. One can verify that $\Phi:=k(\Psi+1)$ is a supersolution of (1.2) for every $k \geq \max \left\{\bar{\xi},\left(\|\Psi\|_{\infty}+1\right)^{p /(1-p)}\right\}$. Indeed, from (1.3) it follows that

$$
\begin{aligned}
L \Phi & \geq k\left(a+\lambda f^{+}\right) \geq k a+\lambda f \geq k^{p}\left(\|\Psi\|_{\infty}+1\right)^{p} a+\lambda f \\
& =a\|\Phi\|_{\infty}^{p}+\lambda f \geq a \Phi^{p}+\lambda f \geq h(x, t, \Phi)+\lambda f
\end{aligned}
$$

in $\Omega \times \mathbb{R}$ and $\Phi=k$ on $\partial \Omega \times \mathbb{R}$.

Let $\lambda>0$, and suppose now that $L^{-1} f \geq 0$. Since $f \not \equiv 0$ we get that $L^{-1} f \not \equiv 0$, and clearly $\lambda L^{-1} f$ is a subsolution of (1.2) because $h(\cdot, \xi) \geq 0$ in $\Omega \times \mathbb{R}$ for all $\xi \geq 0$. Thus, taking into account the above paragraph, [13, Theorem 1] yields some $u_{\lambda}$ solution of (1.2) satisfying $\lambda L^{-1} f \leq u_{\lambda} \leq \Phi$. In particular $u_{\lambda} \in L_{T}^{\infty}$ and hence $u_{\lambda} \in C_{T}$ by Remark 2.1(i). Furthermore, $u_{\lambda}>0$ in $\Omega \times \mathbb{R}$ when $L^{-1} f>0$ in $\Omega \times \mathbb{R}$; and if $L^{-1} f \in P^{\circ}$ and $h(\cdot, \xi) \in L_{T}^{r}$ for some $r>N+2$ and all $\xi \geq 0$, then from Remark 2.1(ii) we find that $u_{\lambda} \in W_{r, T}^{2,1} \cap P^{\circ}$.

On the other hand, let $\lambda_{j} \nearrow \infty$ and suppose there exist nontrivial $u_{\lambda_{j}} \geq 0$ solutions of (1.2) for every $j \in \mathbb{N}$. Let

$$
w_{\lambda_{j}}:=u_{\lambda_{j}} / \lambda_{j}, \quad \xi_{0}(x, t):=\max _{\xi \in[0, \bar{\xi}]} h(x, t, \xi) .
$$

We claim that $\left\|w_{\lambda_{j}}\right\|_{\infty} \leq c$ for some $c>0$ not depending on $j$. Indeed, by (H1) we have that

$$
L w_{\lambda_{j}}=\lambda_{j}^{-1} h\left(x, t, u_{\lambda_{j}}\right)+f \leq \lambda_{j}^{-1} h\left(x, t, \xi_{0}\right)+\lambda_{j}^{-(1-p)} a w_{\lambda_{j}}^{p}+f
$$

in $\Omega \times \mathbb{R}$, and so for all $j$ large enough we obtain that

$$
\left\|w_{\lambda_{j}}\right\|_{L_{T}^{\infty}} \leq\left\|L^{-1}\right\|\left(\left\|h\left(\cdot, \xi_{0}\right)\right\|_{L_{T}^{r}}+\|a\|_{L_{T}^{r}}\left\|w_{\lambda_{j}}\right\|_{L_{T}^{\infty}}^{p}+\|f\|_{L_{T}^{r}}\right)
$$

and the claim is proved. Moreover, this fact and the inequality in (3.2) imply that also $\left\|\lambda_{j}^{-1} h\left(x, t, u_{\lambda_{j}}\right)+f\right\|_{L_{T}^{r}}$ has a bound independent of $j$. Thus, since $L^{-1}: L_{T}^{r} \rightarrow C_{T}$ is 
a compact operator (see Remark 2.1(i)), going to the limit in the equality in (3.2), a standard compactness argument provides some nontrivial $0 \leq w_{\infty} \in C_{T}$ solution of (1.1) with $f$ in place of $g$. Therefore $L^{-1} f \geq 0$ and this concludes the proof.

Theorem 3.2. Let h satisfy (H1) and either (H2) or (H3), let $\xi, b(x, t)$ and $q$ be given by (H2) or (H3) as appropriate, and let $\varphi$ be given by (2.1). Let $0 \equiv f \in L_{T}^{r}$ for some $r>(N+2) / 2$, and suppose $L^{-1} f \nsupseteq 0$.

(i) Then (1.2) has a solution $0<u_{\lambda} \in C_{T}$ for all $\lambda \in(0, \underline{\Lambda}]$, where

$$
\underline{\Lambda}:=C_{b} \inf _{\Omega \times \mathbb{R}} \frac{\varphi}{L^{-1} f^{-}}
$$

with

$$
C_{b}:= \begin{cases}\frac{\beta-\beta^{1 / q}}{\lambda_{1}(b)^{1 /(1-q)}}, \quad \beta:=\min \left\{\underline{\xi} \lambda_{1}(b)^{1 /(1-q)}, q^{q /(1-q)}\right\} & \text { if }(H 2) \text { holds }, \\ \underline{\xi}\left(1-\lambda_{1}(b)\right) & \text { if }(H 3) \text { holds } .\end{cases}
$$

Also, there exists $\Lambda \in[\underline{\Lambda}, \infty)$ such that there is no nonnegative solution for (1.2) if $\lambda>\Lambda$.

Assume, in addition, that either $f \leq 0$ or $\xi \rightarrow h(x, t, \xi)$ is concave for all $\xi \geq 0$ a.e., $(x, t) \in \Omega \times \mathbb{R}$.

(ii1) Then (1.2) has a nontrivial solution $0 \leq u_{\lambda} \in C_{T}$ for all $\lambda \in(0, \Lambda]$.

(ii2) Let $a(x, t)$ and $p$ be given by (H1) and let $\xi_{0}$ be given by (3.1). If $f \leq 0$ then

$$
\Lambda \leq \inf _{\Omega \times \mathbb{R}} \frac{L^{-1}\left[h\left(\cdot, \xi_{0}\right)+\max \left\{1,\left[\left\|L^{-1} h\left(\cdot, \xi_{0}\right)\right\|_{L_{T}^{r}}+\left\|L^{-1}\right\|\|a\|_{L_{T}^{r}}\right]^{p /(1-p)}\right\} a\right]}{L^{-1} f^{-}}
$$

and if $\xi \rightarrow h(\cdot, \xi)$ is concave then $u_{\lambda}$ can be chosen such that $u_{\lambda}>0$ for all $\lambda \in(0, \Lambda)$.

Also, if $h(\cdot, \xi), f \in L_{T}^{r}$ for all $\xi \geq 0$ and some $r>N+2$, then $u_{\lambda} \in W_{r, T}^{2,1}$ whenever such $u_{\lambda}$ exists (in particular, $u_{\lambda} \in P^{\circ}$ whenever $u_{\lambda}>0$ ).

Proof. We proceed in several steps. Let $\lambda \in(0, \underline{\Lambda}]$. We start by constructing a subsolution of (1.2) which is strictly positive in $\bar{\Omega} \times \mathbb{R}$ and belongs to $C_{T}$ for all such $\lambda$ s. Suppose first that $(\mathrm{H} 2)$ is fulfilled. We assume without loss of generality that $b \in L_{T}^{s}$ for some $s>N+2$. So, in particular, $\varphi \in W_{s, T}^{2,1} \cap P^{\circ}$ and the infimum in (3.4) is strictly positive. Let $\beta$ be given by (3.5), and define $\beta_{1}:=\beta \lambda_{1}(b)^{-1 /(1-q)}$ and $v:=\beta_{1} \varphi-\lambda L^{-1} f^{-}$. Observe that $\beta_{1} \leq \xi$ and that $0 \not L^{-1} f^{-} \in C_{T}$ because $L^{-1} f \nsupseteq 0$. It also holds that $0<v \in C_{T}$ since $\lambda \leq \underline{\Lambda}$, (3.4) and (3.5) yield

$$
v \geq \beta_{1} \varphi-\underline{\Lambda} L^{-1} f^{-} \geq\left(\beta_{1}-C_{b}\right) \varphi=\frac{\beta^{1 / q}}{\lambda_{1}(b)^{1 /(1-q)}} \varphi .
$$

Taking into account (1.4), (3.4) and (3.5) and that $\|\varphi\|_{\infty}=1, q \in(0,1)$ and $\lambda \leq \underline{\Lambda}$, after some computations we find that

$$
\begin{aligned}
L v & =\beta_{1} \lambda_{1}(b) b \varphi-\lambda f^{-} \leq \beta_{1} \lambda_{1}(b) b \varphi^{q}+\lambda f \\
& =b \varphi^{q}\left(\beta_{1}-C_{b}\right)^{q}+\lambda f \leq b\left(\beta_{1} \varphi-\underline{\Lambda} L^{-1} f^{-}\right)^{q}+\lambda f
\end{aligned}
$$




$$
\begin{aligned}
& \leq b\left(\beta_{1} \varphi-\lambda L^{-1} f^{-}\right)^{q}+\lambda f \leq h\left(x, t, \beta_{1} \varphi-\lambda L^{-1} f^{-}\right)+\lambda f \\
& =h(x, t, v)+\lambda f
\end{aligned}
$$

in $\Omega \times \mathbb{R}$ and therefore $v$ is a subsolution of (1.2).

If (H3) holds we may argue almost exactly as above. Indeed, if we set $v:=$ $\underline{\xi} \varphi-\lambda L^{-1} f^{-}$then, reasoning as in (3.7), $v \geq\left(\underline{\xi}-C_{b}\right) \varphi=\underline{\xi} \lambda_{1}(b) \varphi>0$ and from (H3) and (3.5) we derive

$$
\begin{aligned}
L v & \leq \underline{\xi} \lambda_{1}(b) b \varphi+\lambda f=b \varphi\left(\underline{\xi}-C_{b}\right)+\lambda f \\
& \leq b\left(\underline{\xi} \varphi-\underline{\Lambda} L^{-1} f^{-}\right)+\lambda f \leq h\left(x, t, \underline{\xi} \varphi-\lambda L^{-1} f^{-}\right)+\lambda f \\
& =h(x, t, v)+\lambda f
\end{aligned}
$$

in $\Omega \times \mathbb{R}$. Therefore, since the first paragraph of the proof of Theorem 3.1 provides supersolutions greater than $k$ for any $k>0$, in both cases we may apply [13, Theorem 1] and obtain a solution $0<u_{\lambda} \in C_{T}$ of (1.2) for every $\lambda \in(0, \underline{\Lambda}]$.

We next define

$$
\Lambda:=\sup \left\{\lambda>0: \text { there exists a solution } u_{\lambda} \geq 0 \text { of (1.2) }\right\} .
$$

By the above paragraph, $\Lambda \geq \underline{\Lambda}$. Moreover, from either (H2) or (H3) we get that $h(\cdot, 0)=0$ and then (since $f \not \equiv 0) u \equiv 0$ cannot be a solution of (1.2) (in fact, it cannot be a subsolution because $\left.L^{-1} f \nsupseteq 0\right)$. Hence, Theorem 3.1 says that $\Lambda<\infty$.

Let us prove (ii1). Consider first $\lambda \in(0, \Lambda)$. Recalling the beginning of the proof of Theorem 3.1, in order to prove the existence of a nonnegative solution for (1.2) it is enough to supply a nonnegative subsolution. Suppose that $f \leq 0$ and choose $\bar{\lambda} \in(\lambda, \Lambda)$ such that there exists $u_{\bar{\lambda}} \geq 0$ solving (1.2) with $\bar{\lambda}$ in place of $\lambda$. Then clearly $u_{\bar{\lambda}}$ is a subsolution. Suppose now that $\xi \rightarrow h(\cdot, \xi)$ is concave. By the first part of the proof we can take $\underline{\lambda} \in(0, \lambda)$ such that there exists a subsolution $u_{\underline{\lambda}}>0$ for (1.2). Let $u_{\bar{\lambda}}$ be as above, and set $s:=(\lambda-\underline{\lambda}) /(\bar{\lambda}-\underline{\lambda})$ and $w_{\lambda}:=s u_{\bar{\lambda}}+(1-s) u_{\underline{\lambda}}$. We have

$$
\begin{aligned}
L w_{\lambda} & \leq s\left(h\left(x, t, u_{\bar{\lambda}}\right)+\bar{\lambda} f\right)+(1-s)\left(h\left(x, t, u_{\lambda}\right)+\lambda f\right) \\
& =s h\left(x, t, u_{\bar{\lambda}}\right)+(1-s) h\left(x, t, u_{\underline{\lambda}}\right)+(s \bar{\lambda}+(1-s) \underline{\lambda}) f \\
& \leq h\left(x, t, s u_{\bar{\lambda}}+(1-s) u_{\underline{\lambda}}\right)+\lambda f=h\left(x, t, w_{\lambda}\right)+\lambda f
\end{aligned}
$$

in $\Omega \times \mathbb{R}$ and so $w_{\lambda}>0$ is a subsolution for (1.2). Furthermore, note that the second assertion of (ii2) also follows from (3.8).

We prove next the existence of nonnegative solution for $\lambda=\Lambda$. We proceed in a similar way to the final part of the proof of Theorem 3.1. Let $\lambda_{j} \nearrow \Lambda$ and let $u_{\lambda_{j}} \geq 0$ be the corresponding solutions of (1.2) found by the above paragraph, and let $\xi_{0}$ be given by (3.1). From (H1) we have for all $j$ that

$$
L u_{\lambda_{j}}=h\left(x, t, u_{\lambda_{j}}\right)+\lambda_{j} f \leq h\left(x, t, \xi_{0}\right)+a u_{\lambda_{j}}^{p}+\Lambda f
$$

in $\Omega \times \mathbb{R}$ and hence, arguing as in (3.3), we see that $\left\|u_{\lambda_{j}}\right\|_{L_{T}^{\infty}} \leq c$ for some $c>0$ not depending on $j$. Passing to the limit in the equality in (3.9), again by compactness we obtain some nontrivial $u_{\infty} \geq 0$ solving (1.2) for $\lambda=\Lambda$. 
Let us prove (3.6). Suppose that $f \leq 0$. Notice first that the infimum in (3.6) is strictly positive because, for instance, $L^{-1} a \geq L^{-1}\left(a \chi_{\{a \leq 1\}}\right) \in P^{\circ}$. Observe next that if $u$ is a solution of (1.2) then in view of (H1), $L u \leq h\left(x, t, \xi_{0}\right)+a u^{p}$ in $\Omega \times \mathbb{R}$ and therefore

$$
\|u\|_{L_{T}^{\infty}} \leq \max \left\{1,\left[\left\|L^{-1} h\left(\cdot, \xi_{0}\right)\right\|_{L_{T}^{r}}+\left\|L^{-1}\right\|\|a\|_{L_{T}^{r}}\right]^{1 /(1-p)}\right\}:=M .
$$

Thus, (1.2) and (H1) imply that $0 \leq u \leq L^{-1}\left(h\left(\cdot, \xi_{0}\right)+M^{p} a\right)-\lambda L^{-1} f^{-}$in $\Omega \times \mathbb{R}$, which in turn yields (3.6).

To conclude the proof we note that the final statement follows as in Theorem 3.1 from Remark 2.1.

Remark 3.3. Let us mention that if $h$ satisfies (H1), (H2) or (H3), and $\xi \rightarrow h(\cdot, \xi)$ is concave for all $\xi \geq 0$, then as a consequence of the proof of Theorem 3.2 the solution $u_{\lambda}$ provided by Theorem 3.1 can be chosen satisfying $u_{\lambda}>0$ in $\Omega \times \mathbb{R}$ for every $\lambda>0$ by requiring only that $L^{-1} f \geq 0$ (in particular, under these assumptions, $u_{\lambda} \in W_{r, T}^{2,1} \cap P^{\circ}$ whenever $h(\cdot, \xi), f \in L_{T}^{r}$ for some $\left.r>N+2\right)$. Indeed, a quick look at the paragraph containing (3.8) shows that it suffices to proceed exactly as there.

Remark 3.4. Suppose that $h$ fulfills (H3) with equality holding in (1.5), and assume also that $\sup _{\xi>0} \xi^{-1} h(\cdot, \xi)=\lim _{\xi \rightarrow 0^{+}} \xi^{-1} h(\cdot, \xi)$ (let us point out that this last hypothesis occurs, for instance, in logistic-type nonlinearities). Then the condition $\lambda_{1}(b)<1$ in (H3) is necessary for the existence of nonnegative solutions for (1.2) in the case $f \leq 0$ in $\Omega \times \mathbb{R}$. Indeed, if $u$ is such a solution,

$$
L u<h(x, t, u) \leq u \sup _{\xi>0} \frac{h(\cdot, \xi)}{\xi}=u \lim _{\xi \rightarrow 0^{+}} \frac{h(\cdot, \xi)}{\xi}=b u
$$

in $\Omega \times \mathbb{R}$ and hence Remark 2.2(ii) tells us that $\lambda_{1}(b)<1$.

\section{References}

[1] J. Ali, R. Shivaji and K. Wampler, 'Population models with diffusion, strong Allee effect and constant yield harvesting', J. Math. Anal. Appl. 352 (2009), 907-913.

[2] W. Allegretto and P. Nistri, 'Existence and stability for nonpositone elliptic problems', Nonlinear Anal. 23 (1994), 1243-1253.

[3] W. Allegretto, P. Nistri and P. Zecca, 'Positive solutions of elliptic nonpositone problems', Differential Integral Equations 5 (1992), 95-101.

[4] S. Carl, 'Multiple solutions of quasilinear periodic-parabolic inclusions', Nonlinear Anal. 72 (2010), 2909-2922.

[5] A. Castro, C. Maya and R. Shivaji, 'Nonlinear eigenvalue problems with semipositone structure', Proc. Conf. on Nonlinear Differential Equations, Coral Gables, FL, 1999 (Southwest Texas State University, Texas, 2000), Electron. J. Differ. Equ. Conf. 5 (2000), 33-49.

[6] A. Castro and R. Shivaji, 'Positive solutions for a concave semipositone Dirichlet problem', Nonlinear Anal. 31 (1998), 91-98.

[7] D. Costa, H. Tehrani and J. Yang, 'On a variational approach to existence and multiplicity results for semipositone problems', Electron. J. Differential Equations 2006, No. 11, 10p.

[8] Q. Dai and Y. Gu, 'Positive solutions for non-homogeneous semilinear elliptic equations with data that changes sign', Proc. Roy. Soc. Edinburgh Sect. A 133 (2003), 297-306. 
[9] Q. Dai and L. Peng, 'Necessary and sufficient conditions for the existence of nonnegative solutions of inhomogeneous p-Laplace equation', Acta Math. Sci. Ser. B Engl. Ed. 27 (2007), 34-56.

[10] Q. Dai and J. Yang, 'Positive solutions of inhomogeneous elliptic equations with indefinite data', Nonlinear Anal. 58 (2004), 571-589.

[11] E. N. Dancer and J. Shi, 'Uniqueness and nonexistence of positive solutions to semipositone problems', Bull. Lond. Math. Soc. 38 (2006), 1033-1044.

[12] D. Daners and P. Koch-Medina, Abstract Evolution Equations, Periodic Problems and Applications, Pitman Research Notes in Mathematics Series, 279 (Longman Scientific \& Technical, 1992).

[13] J. Deuel and P. Hess, 'Nonlinear parabolic boundary value problems with upper and lower solutions', Israel J. Math. 29 (1978), 92-104.

[14] P. Girão and H. Tehrani, 'Positive solutions to logistic type equations with harvesting', J. Differential Equations 247 (2009), 574-595.

[15] T. Godoy and U. Kaufmann, 'On principal eigenvalues for periodic parabolic problems with optimal condition on the weight function', J. Math. Anal. Appl. 262 (2001), 208-220.

[16] T. Godoy and U. Kaufmann, 'On positive solutions for some semilinear periodic parabolic eigenvalue problems', J. Math. Anal. Appl. 277 (2003), 164-179.

[17] T. Godoy and U. Kaufmann, 'On the existence of positive solutions for periodic parabolic sublinear problems', Abstr. Appl. Anal. 2003 (2003), 975-984.

[18] T. Godoy and U. Kaufmann, 'On some semilinear periodic parabolic problems', Rend. Istit. Mat. Univ. Trieste 38 (2006), 139-148.

[19] T. Godoy and U. Kaufmann, 'Periodic parabolic problems with nonlinearities indefinite in sign', Publ. Mat. 51 (2007), 45-57.

[20] P. Hess, Periodic-Parabolic Boundary Value Problems and Positivity, Pitman Research Notes in Mathematics Series, 247 (Longman Scientific \& Technical, 1991).

[21] N. Hirano and W. Kim, 'Multiple existence of periodic solutions for a nonlinear parabolic problem with singular nonlinearities', Nonlinear Anal. 54 (2003), 445-456.

[22] O. Ladysženkaja, V. Solonnikov and N. Ural'ceva, Linear and Quasilinear Equations of Parabolic Type, Translations of Mathematical Monographs, 23 (American Mathematical Society, Providence, RI, 1968).

[23] G. Lieberman, 'Time-periodic solutions of linear parabolic differential equations', Comm. Partial Differential Equations 24 (1999), 631-664.

[24] T. Ouyang and J. Shi, 'Exact multiplicity of positive solutions for a class of semilinear problem, II', J. Differential Equations 158 (1999), 94-151.

\section{T. GODOY, FaMAF, Universidad Nacional de Córdoba, (5000) Córdoba, Argentina \\ e-mail: godoy@mate.uncor.edu}

U. KAUFMANN, FaMAF, Universidad Nacional de Córdoba, (5000) Córdoba, Argentina

e-mail: kaufmann@mate.uncor.edu 